\section{ORIGINAL RESEARCH}

C.M. Phan

A.J. Yoo

J.A. Hirsch

R.G. Nogueira

R. Gupta

\title{
Differentiation of Hemorrhage from lodinated Contrast in Different Intracranial Compartments Using Dual-Energy Head CT
}

BACKGROUND AND PURPOSE: Identification of $\mathrm{ICH}$, particularly after ischemic stroke therapy, is important for guiding subsequent antithrombotic management and is often confounded by contrast staining or extravasations within intracerebral or extra-axial compartments. This study evaluates the accuracy of DECT in distinguishing $\mathrm{ICH}$ from iodinated contrast in patients who received contrast via IA or IV delivery.

MATERIALS AND METHODS: Forty patients who had received IA or IV contrast were evaluated using a DECT scanner at $80 \mathrm{kV}$ and $140 \mathrm{kV}$ to distinguish hyperdensities secondary to contrast staining or extravasation from those representing $\mathrm{ICH}$. A 3-material decomposition algorithm was used to obtain virtual noncontrast images and iodine overlay images. Sensitivity, specificity, and accuracy of DECT in prospectively distinguishing intracranial contrast from hemorrhage within parenchymal, subarachnoid, extra-axial, intraventricular, and intra-arterial compartments were computed using routine clinical follow-up imaging as the standard of reference.

RESULTS: A total of 148 foci of intracranial hyperattenuation were identified. Of these, 142 were correctly classified for the presence of hemorrhage by DECT. The sensitivity, specificity, and accuracy for identifying hemorrhage, depending on the compartment being considered, were $100 \%, 84.4 \%-$ $100 \%$, and $87.2 \%-100 \%$, respectively. The only instances where DECT failed to correctly identify the source of hyperattenuation was in the presence of diffuse parenchymal calcification $(n=5)$ and a metallic streak artifact $(n=1)$.

CONCLUSION: After IA and/or IV contrast administration, DECT can accurately differentiate all types of $\mathrm{ICH}$ from iodinated contrast without employing any additional radiation.

ABBREVIATIONS: $\mathrm{BP}=$ brain parenchyma; $\mathrm{CNR}=$ contrast-to-noise ratio; $\mathrm{DECT}=$ dual-energy $\mathrm{CT}$ $\mathrm{HU}=$ Hounsfield unit; $\mid \mathrm{A}=$ intra-arterial; $\mid \mathrm{AT}=$ intra-arterial therapy; $\mathrm{ICH}=$ intracranial hemorrhage; $\mathrm{SE}=$ single energy; $\mathrm{VNC}=$ virtual noncontrast

S taining of brain parenchyma and blood vessels is a wellecognized phenomenon of IA contrast administration. ${ }^{1}$ Furthermore, extravasation of contrast into the subarachnoid space is fairly commonly seen following intra-arterial stroke therapy with mechanical devices. These phenomena can also be seen with IV contrast administration. For example, both intratumoral hemorrhage and contrast enhancement are common. Hemorrhagic transformation and gyriform enhancement of subacute stroke are well described, and distinguishing them is clinically important. When contrast is administered in the setting of intracranial hemorrhage, petechial extravasation of contrast in this hemorrhagic bed is a wellrecognized phenomenon and has been dubbed the "spot sign."

On a routine noncontrast head CT scan performed after IA

Received July 10, 2011; accepted after revision August 29

From the Department of Radiology (C.M.P., A.J.Y., J.A.H., R.G.), Massachusetts General Hospital, Boston, Massachusetts; Departments of Neurology, Neurosurgery, and Radiology (R.G.N.), Emory University School of Medicine, Marcus Stroke \& Neuroscience Center, Grady Memorial Hospital, Atlanta, Georgia; Department of Neurology (R.G.N.), Massachusetts General Hospital, Harvard Medical School, Wang Ambulatory Care Center, Boston, Massachusetts.

C.M.P. and A.J.Y. contributed equally to this article.

Please address correspondence to Catherine M. Phan, Department of Radiology, Massachusetts General Hospital, 55 Fruit St, Neuroradiology GRB-273A, Boston, MA 02114; e-mail: cmphan@partners.org

Indicates article with supplemental on-line tables.

http://dx.doi.org/10.3174/ajnr.A2909 or IV contrast administration, it can be difficult to differentiate a hyperattenuation resulting from iodinated contrast versus that arising from intracranial hemorrhage. ${ }^{2}$ This differentiation is especially critical in the setting of acute stroke or trauma, when antithrombotic therapy is being considered. The current standard of care for such discrimination is repeat follow-up imaging ${ }^{1}$ : Contrast staining generally washes out within 24-48 hours, while hemorrhage persists for days to weeks.

Early results have shown that DECT can distinguish hemorrhage from iodinated contrast. ${ }^{3-5}$ This discrimination is based on the differences between the photoelectric and Compton scattering components underlying the $\mathrm{x}$-ray attenuation of hemorrhage and iodine. Because both phenomena are dependent on the $x$-ray photon energy, one can discriminate the pixel attenuation arising from these 2 effects by scanning at 2 different energy levels, such as $80 \mathrm{kV}$ and $140 \mathrm{kV}$. Assuming that there can only be hemorrhage and/or iodine (in addition to water and tissue), this information can be used to determine the amount of each material present in each voxel.

In this research, we considered a mixed cohort after both IA and IV contrast administration. The overall goal was to establish the general hypothesis that DECT is useful in all settings where one has to distinguish between ICH and iodine from prior contrast administration, irrespective of how the contrast was administered. The sensitivity, specificity, and accuracy of DECT in differentiating hemorrhage from iodinated contrast 
were assessed for the intraparenchymal, subarachnoid, extraaxial, intraventricular, and intra-arterial compartments.

\section{Materials and Methods}

\section{Patient Selection}

The patients were prospectively screened and retrospectively analyzed. The determination of which patients were routed to the DECT scan was based on the following criteria: 1) all patients who received IAT after an acute stroke, and 2) patients who received IV contrast for any reason (eg, carotid stent placement, tumor evaluation, or trauma), where a question of hemorrhage versus contrast was raised by an SE energy scan.

This study was approved by the local institutional review board (IRB Protocol \#2008P002351). The requirement for informed consent for the retrospective analysis was waived by the IRB. From October 2008 to May 2010, 40 patients (mean age 64.9 years; range 28-94) were referred for a DECT scan and were included in the analysis. There were 27 men (mean age 63.1 years; range 28 -94) and 13 women (mean age 68.7 years; range 43-84). The study cohort included 20 acute stroke patients, of whom 18 underwent IAT; 15 patients evaluated for carotid stenosis; 3 patients evaluated for trauma; and 2 patients evaluated for tumor assessment. At our institution, all stroke patients who undergo conventional angiography for intra-arterial embolectomy or thrombolysis are evaluated by using a postprocedure noncontrast CT for any complications. Therefore, this cohort constituted the largest subpopulation of patients scanned by DECT. All IAT patients were scanned within 30 minutes of the end of the interventional procedure.

It should be noted that our cohort of patients includes 15 cases with suspected carotid stenosis and old strokes. These patients underwent a DECT angiogram that included a dual-energy head CT. These cases do not contribute to the hyperattenuated lesions that were evaluated and therefore do not affect the measured sensitivity and specificity of the DECT technique. They do, however, prove that the presence of contrast in the intravascular space at DECT does not artificially create any hyperattenuated artifacts that can be confused as intracranial hemorrhage.

Patients undergoing DECT imaging were retrospectively analyzed based on the availability of follow-up imaging to establish the status of each observed hyperattenuating focus. Three patients were excluded from analysis due to lack of follow-up imaging. Two patients underwent a dual-energy evaluation to further assess a new intraparenchymal hyperattenuation that was observed on a routine NCCT scan.

\section{DECT Scanning}

Somatom Definition (Siemens Healthcare, Forchheim, Germany) was used for dual-energy scanning. This scanner consists of $2 \mathrm{x}$-ray tubes and 2 detectors, mounted on a common gantry. In the dualenergy mode, the tube voltage and tube current settings for each $\mathrm{x}$-ray source are set and optimized independently. The simultaneous acquisition of data at 2 energies in a single scan also minimizes misregistration and motion artifacts. The dual-energy technique is based on the behavior of various materials when exposed to $\mathrm{x}$-rays at low and high energy, leading to differences in attenuation. These differences reflect the energy and material dependency of Compton and photoelectric effects. $^{6}$

CT examinations were performed using the following protocol: Tube A at $80 \mathrm{kV}, 499 \mathrm{~mA}$; Tube B at $140 \mathrm{kV}, 118 \mathrm{~mA}$ (effective mAs of 714 and 168, respectively); and a collimation of $14 \times 1.2 \mathrm{~mm}$. These settings split the overall dose nearly equally between the 2 imaging chains. The total effective dose was similar to that in a conventional head CT (approximately $3 \mathrm{mSv}$ ). The average CT dose index-volume for a dual-energy head scan was $66 \mathrm{mGy}$, which is quite similar to that for a single-energy conventional head CT. It is also well within the American College of Radiology guidelines of $80 \mathrm{mGy}$.

The projection data acquired at 80 and $140 \mathrm{kV}$ were reconstructed separately at 4.0-mm section thickness using an H30 kernel (medium smooth), generating 3 sets of images: an $80-\mathrm{kV}$ image, a $140-\mathrm{kV}$ image, and an SE image. The SE image set, which is a weighted sum of the $80-\mathrm{kV}$ and $140-\mathrm{kV}$ images, simulates an equivalent $120-\mathrm{kV}$ image; it has a higher signal intensity-to-noise ratio than the constituent 80 and $140-\mathrm{kV}$ image sets.

Dual-energy postprocessing was performed using dedicated software (Syngo Dual Energy Brain Hemorrhage, Siemens): Images were reconstructed at $1.5-\mathrm{mm}$ section thickness, with $0.7-\mathrm{mm}$ increments (D30s kernel). The analysis of low- and high-energy images ${ }^{7,8}$ was performed using a 3-material decomposition algorithm based on brain parenchyma, hemorrhage, and iodine, producing a VNC and an iodine-only overlay image. DECT allows one to split every voxel in the acquired $(80 \mathrm{kV}, 140 \mathrm{kV})$ image pair along any 2 preselected base materials. In this application, the 2 base materials were BP and ICH. If each voxel only consisted of these 2 materials, its measured attenuation would be a linear combination of the attenuation values of BP and $\mathrm{ICH}$, both at $80 \mathrm{kVp}$ and $140 \mathrm{kVp}$. To the extent the measured value of a voxel does not conform to a mixture of the 2 base materials, the difference can be attributed to a third preselected material. In the current application, the third material chosen was iodine. The offset from the linear combination of ICH and BP represents the amount of iodinated contrast present in a voxel. The iodine-only images display this offset, and the VNC images display a combination of BP and ICH values. It should be noted that DECT is limited to 3-material decomposition; this limitation is based on the underlying principle that only 2 independent physical processes (photoelectric and Compton effects) are relevant for the spectrum of $\mathrm{x}$-ray energies used in clinical CT.

\section{Image Analysis}

The images were reviewed by 3 experienced radiologists with 8 (R.G.), 8 (C.M.P.), and 9 (A.J.Y.) years of experience. Image analysis was performed by consensus. All intracranial hyperdensities on the simulated $120-\mathrm{kV}$ image were classified as intraparenchymal, subarachnoid, extra-axial, intraventricular, and intra-arterial. This last category was added to distinguish vessel wall staining from intra-arterial, hyperattenuated thrombus. Such a distinction, if it can be made on a noncontrast DECT scan, can potentially differentiate successful recanalization and vessel wall staining after IAT from persistent thrombus.

All the hyperattenuated lesions were prospectively analyzed and classified as hemorrhage, contrast, or a combination of the 2, based on the $\mathrm{VNC}$ and iodine overlay images. A hyperattenuation only seen on the VNC image was classified as hemorrhage; a hyperattenuation only seen on the iodine overlay image was deemed as contrast. In 3-material decomposition, an area of calcification would result in hyperattenuation on both VNC and iodine overlay images. This can potentially be confused with a combination of hemorrhage and contrast. With calcification, however, the pattern of hyperattenuation on VNC image will closely mirror that on iodine overlay image; a combination of hemorrhage and contrast staining would not follow this pattern, as their distribution is not expected to mirror each other exactly. 
Imaging analysis for each case was completed before the follow-up imaging became available, and an appropriate report was dictated. A contiguous sulcal hyperattenuation was counted as one focus of subarachnoid hemorrhage. Each cistern (ie, perimesencephalic cistern, basilar cistern, Sylvian fissure, suprasellar cistern) was counted as a separate focus of subarachnoid hemorrhage.

The ground truth in each case was determined by using the follow-up images from either noncontrast CT scanning or MR imaging. A washout or near-complete clearing of the hyperattenuation in 24-48 hours on a SE noncontrast CT scan was used as evidence for contrast staining. ${ }^{1,9-11}$ If it persisted for longer than 48 hours, and developed a characteristic rim of hypoattenuation (presumed to be edema or infarct), then this hyperattenuation was classified as containing hemorrhage. The 48-hour time cutoff is what we routinely use in our clinical practice. The choice of a SECT as the follow-up scan was made to stay within the standard imaging techniques for the reference standard. When a gradient-echo $\mathrm{T} 2{ }^{\star}$-weighted MR image was available, the presence of decreased signal intensity in the corresponding area of hyperattenuation was taken as the reference standard.

\section{Statistical Analysis}

The presence or absence of hemorrhage in the various intracranial compartments on the DECT images and on the follow-up images (the "gold standard") was statistically analyzed to determine the accuracy of DECT. Pure hemorrhage or mixed hemorrhage with iodine was considered as positive for hemorrhage on DECT images. The following definitions were used:

True-positive: A hyperattenuation on the VNC image that persists on the follow-up CT and/or shows susceptibility on a follow-up MR imaging.

False-positive: A hyperattenuation on the VNC image that shows near complete washout on the follow-up CT and/or has no susceptibility on a follow-up MR imaging.

True-negative: A hyperattenuation on the iodine overlay image without a concomitant hyperattenuation on the VNC image that shows near complete washout on the follow-up CT and/or has no susceptibility on a follow-up MR imaging.

False-negative: A hyperattenuation on the iodine overlay image without a concomitant hyperattenuation on the VNC image that shows persistent hyperattenuation on the follow-up CT and/or has susceptibility on a follow-up MR imaging.

These values were used to derive the sensitivity, specificity, and accuracy of DECT using MedCalc 10.0 software (Mariakerke, Belgium).

It should be emphasized that our method of lesion counting is methodologically sound. To determine how sensitive and specific the DECT technique is in differentiating hemorrhage from iodinated contrast, we need to present varied and multiple concentrations of these materials in different intracranial spaces. The hyperattenuated foci also need to be presented in different infra- and supratentorial locations so that the effect of various CT artifacts such as beam hardening, streak, partial volume averaging, and spiral windmill are properly represented in the final aggregate statistics regarding sensitivity and specificity. Therefore, the basis for such statistical computation should be the different number of hyperattenuated foci that were evaluated by the technique. It is true that in any 1 patient, the etiology of different, noncontiguous foci is likely to be the same. However, the question being asked is, can DECT determine whether a given hyperattenuation is arising from hemorrhage or contrast irrespective of concentration and artifact pattern? Therefore, it is fair to use different noncontiguous foci of hyperdensities as the unit of counting. For subarachoid space, noncontiguity was the main criterion; we did not count each sulcus separately. In cisternal spaces, the entire cistern was considered as 1 unit of hyperattenuation representing a unique instance of the underlying process responsible for the hyperattenuation.

\section{Results}

\section{Findings and Analysis}

One-hundred-forty-eight foci of intracranial hyperattenuation were identified on the SE images. According to the DECT image interpretation, areas of hyperattenuation were prospectively classified as iodinated contrast staining only $(n=70$; $47.3 \%)$, hemorrhage only $(n=31 ; 20.9 \%)$, or mixed contrast and hemorrhage $(n=47 ; 31.8 \%$; On-line Table 1$)$.

All 70 hyperdensities deemed by DECT as contrast only were demonstrated to have no hemorrhage (On-line Table 1) by complete washout, lack of susceptibility, or both on follow-up imaging. These cases of pure iodinated contrast staining or extravasation were identified in the following locations: intraparenchymal $(n=33 ; 47.1 \%)$, subarachnoid $(n=20$; $28.6 \%)$, intra-arterial $(n=13 ; 18.6 \%)$, and intraventricular $(n=4 ; 5.7 \%)$. All 31 hyperattenuated foci deemed by DECT to have hemorrhage only were confirmed as such on subsequent imaging. These were classified as intraparenchymal $(n=5$; $16.1 \%)$, subarachnoid ( $n=6 ; 19.4 \%)$, subdural or epidural $(n=8 ; 25.8 \%)$, intra-arterial $(n=10 ; 32.3 \%)$, and intraventricular $(n=2 ; 6.4 \%)$. In the 47 cases that were classified as mixed contrast and hemorrhage, 41 were found to have a hemorrhagic component. In 5 cases, there were areas of parenchymal calcifications, as determined by available prior imaging. Metallic streak artifact $(n=1)$ was visible on both VNC image and iodine overlay image.

The overall sensitivity was $100 \%(94.9 \%-100 \%)$ and specificity was $92.8 \%(83.9-97.6 \%)$ for the presence of hemorrhage. The sensitivities and specificities for the various intracranial compartments are listed in On-line Table 2. The specificities ranged from $84.4 \%-100 \%$, and the accuracy from $87.2 \%-100 \%$, depending on the compartment being considered.

In the following, the different types of hyperattenuation are presented by intracranial compartment.

\section{Intraparenchymal Contrast Staining}

Forty areas of intraparenchymal contrast staining were identified on the iodine overlay images, either in isolation $(n=33)$ or in association with a hyperattenuation on the VNC image $(n=7)$. Thirty-three of 40 areas $(82.5 \%)$ that were present only on the iodine overlay images demonstrated gradual washout on follow-up imaging, confirming the purported etiology (Fig 1). The other 7 areas (17.5\%) that were hyperattenuated on both VNC and iodine overlay images demonstrated partial washout on 24-48 hour follow-up imaging, suggesting hemorrhage or calcification superimposed on the top of contrast staining.

\section{Intraparenchymal Hemorrhage}

In this cohort of patients, 5 areas of intraparenchymal hyperattenuation were deemed to be secondary to pure hem- 


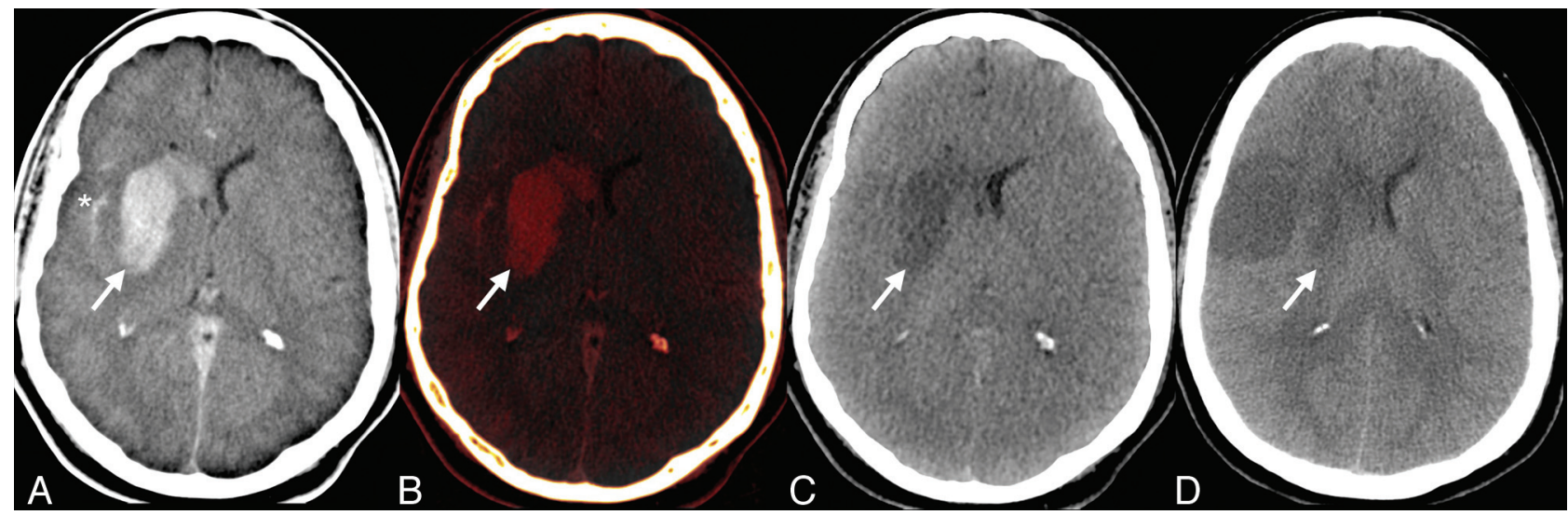

Fig 1. Intraparenchymal and subarachnoid foci of hyperattenuation due to iodinated contrast staining of infarcted brain parenchyma in a 78-year-old woman with recanalization of the right terminal internal carotid artery. $A$, Intraparenchymal hyperattenuation is seen in the right basal ganglia (arrow) and in the subarachnoid space ${ }^{*}$ ) on the SE image. $B$, These foci correspond to areas of diffuse contrast staining on the iodine overlay image. $C$, VNC image shows an area of hypoattenuation related to the infarct (arrow). $D$, Follow-up NCCT demonstrates complete washout of the contrast in both locations.

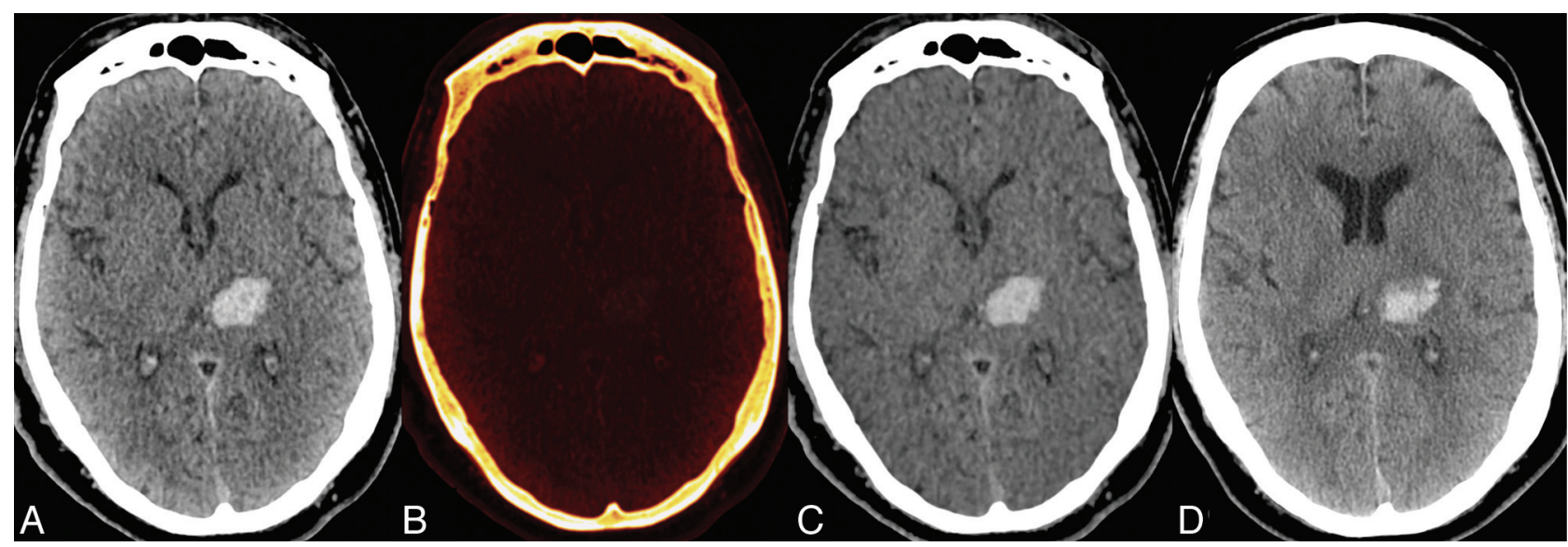

Fig 2. Left thalamic intraparenchymal hyperattenuation due to hemorrhage of uncertain etiology in a 51-year-old man referred for altered mental status. The patient underwent a dual energy CTA and MR imaging for further evaluation. $A$, SE image shows left thalamic intraparenchymal hyperattenuation without corresponding hyperattenuation on the iodine overlay image $(B)$. $C$, The focus of hyperattenuation is well demonstrated on the VNC image. A 24-hour follow-up NCCT scan (D) demonstrates largely stable hyperattenuation in the left thalamus, with an increase in the surrounding edema, confirming the original diagnosis of intraparenchymal hemorrhage.

orrhage, as there was corresponding hyperattenuation on VNC images; lack of any residual hyperattenuation on the iodine overlay images suggested that there was no component arising from contrast staining. Subsequent CT scanning showed persistent hyperattenuation, with increasing surrounding edema, consistent with hemorrhagic conversion of infarcted brain parenchyma. Intraparenchymal contrast staining would have substantially decreased in attenuation due to washout on the follow-up study. Figure 2 shows an example of this category.

\section{Subarachnoid Contrast Extravasation}

Twenty contiguous areas of subarachnoid contrast staining were identified by DECT and were confirmed by subsequent imaging. Fig 3 illustrates an example of diffuse hyperattenuation in the right Sylvian fissure (arrow) on the SE image (Fig $3 A$ ), which matched the subarachnoid hyperattenuation on the iodine overlay image (Fig $3 B$ ). The lack of a corresponding hyperattenuation on the VNC image (Fig 3C) suggested contrast extravasation in the Sylvian fissure. This was confirmed by a 24-hour follow-up NCCT (Fig 3D), where the focus of hyperattenuation had nearly completely resolved. A blood clot within the same area would have taken much longer to resolve.

\section{Subarachnoid Hemorrhage}

Six of the 37 hyperattenuated subarachnoid areas (16.21\%) were identified on the VNC images but were not present on the iodine overlay images. These were confirmed to be areas of subarachnoid hemorrhage by repeat imaging within 24 hours. An example is shown in Fig 4 in a patient with posttraumatic SAH. There were multiple foci of hemorrhage in this case. While some of the foci were clearly posttraumatic, others were more ambiguous. In such cases, our protocol is to perform a dual-energy CTA to rule out an underlying vascular malformation, aneurysm, or vasculitis.

\section{Mixed Subarachnoid Hemorrhage and Contrast Extravasation}

A noncontrast enhanced CT scan immediately after intra-arterial conventional angiography and embolectomy procedure in 4 patients demonstrated diffuse subarachnoid hyperattenuation on the SE image. There were some areas that were denser 


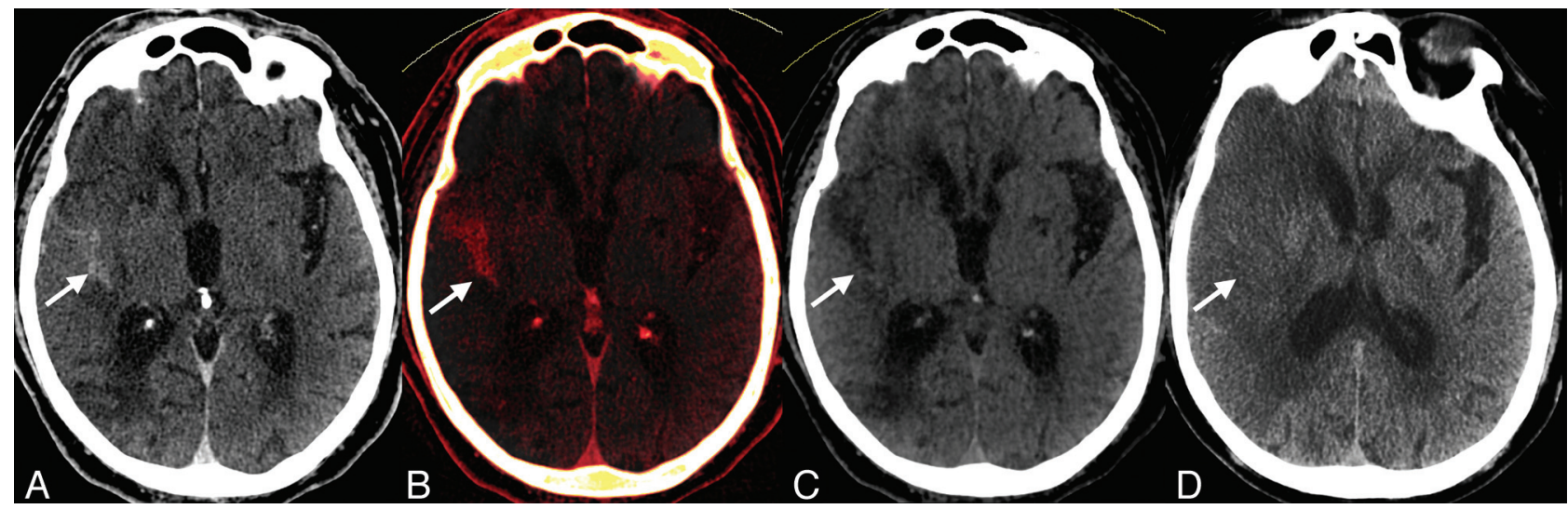

Fig 3. Subarachnoid hyperattenuation due to contrast staining in a 79-year-old man treated endovascularly for an acute stroke in the right MCA territory. $A$, Diffuse hyperattenuation in the right Sylvian fissure (arrow) on the SE image corresponds to the hyperattenuation seen on the iodine overlay image $(B)$. The lack of hyperattenuation on the VNC image $(C)$ suggests contrast extravasation in the Sylvian fissure. This is confirmed by the near-complete washout of the hyperattenuation on the 24-hour follow-up NCCT (D).

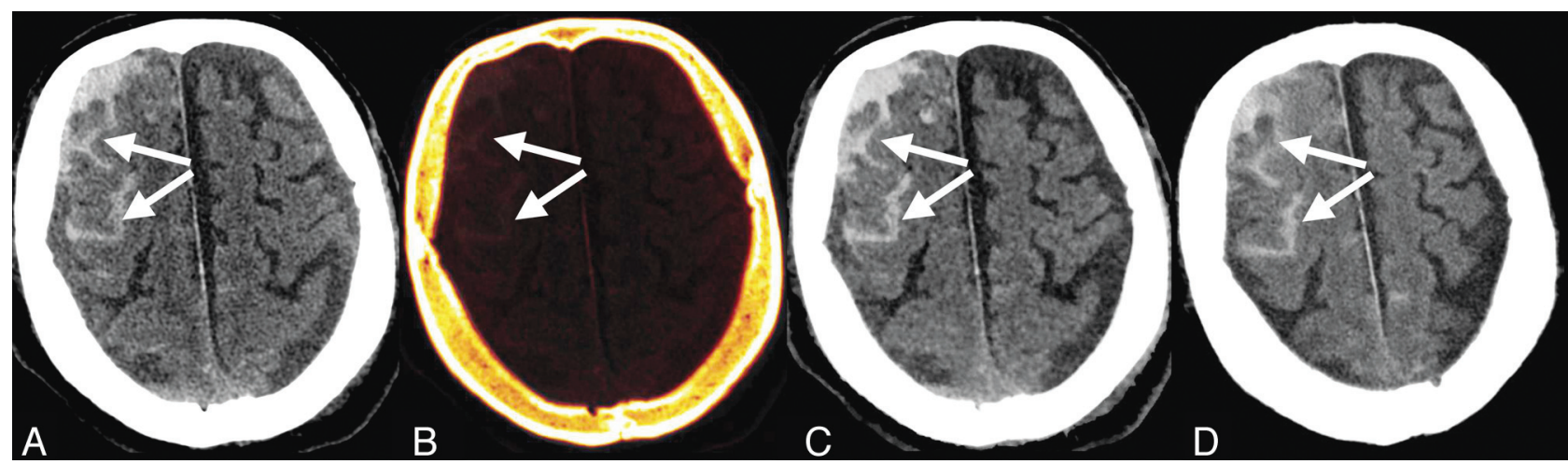

Fig 4. Subarachnoid hyperattenuation due to hemorrhage in a 64-year-old man. $A$, There are foci of sulcal hyperattenuation (arrows) on the SE image. $B$, No corresponding hyperattenuation is seen on the iodine overlay image. $C$, VNC image shows identical foci of sulcal hyperattenuation, suggesting subarachnoid hemorrhage that was confirmed by the 24-hour follow-up NCCT (D).

( $n=11)$ than others, raising the suspicion for subarachnoid hemorrhage superimposed on diffuse subarachnoid contrast extravasation. Mixed hemorrhage and contrast was confirmed by follow-up imaging (Fig 5).

\section{Extra-Axial Hemorrhage}

Eight extra-axial hemorrhages only seen on VNC images were detected by DECT and were confirmed by subsequent imaging.

\section{Intra-Arterial Thrombus and Contrast Staining}

Forty-nine cases of intra-arterial hyperattenuation were identified on the SE images. DECT classified 26 of these cases as intra-arterial contrast staining; 13 cases were identified as a mixture of intra-arterial thrombus associated with contrast staining. The remaining 10 instances were those of pure thrombus without any contrast staining, either in the adjacent vessel wall or in the thrombus itself.

\section{Intraventricular Hyperattenuation}

Seven cases of intraventricular hyperattenuation were identified on the SE images. DECT correctly determined these to be contrast staining only $(n=4 ; 57.15 \%)$, hemorrhage only $(n=$ $2 ; 28.6 \%)$, or mixed hemorrhage and contrast staining $(n=1$; $14.29 \%)$.

\section{Discussion}

This study demonstrates that DECT scanning can prospectively differentiate intracranial hemorrhage from iodinated contrast staining and extravasation in all intracranial compartments with high accuracy. The discriminatory power of DECT comes at no extra cost in terms of radiation dose to the patient and does not compromise image quality compared with the traditional SE NCCT. The radiation dose from DECT head scan (average CT dose index-volume $=66 \mathrm{mGy}$ ) is comparable with that from SE head scan. It is possible to achieve this on a dual-source CT because the tube current on each source can be individually optimized. The protocol used in this study split the total dose approximately equally between the $2 \mathrm{x}$-ray sources so that the overall scan is dose-matched with a conventional head CT.

While the current study was not designed for comparison of image quality between single- and dual-energy CT, our experience shows that the image quality of a virtual single-energy image obtained from dual-source, dual-energy CT is comparable to an image from a single-source, single-energy CT. The iodine overlay image and the virtual noncontrast image derived from DECT, which represent less than full dose, are noisier. However, they have sufficient image quality for differentiating hemorrhage from iodine. Prior studies have reported similar results. For example, Ferda et $\mathrm{al}^{4}$ evaluated intracranial 


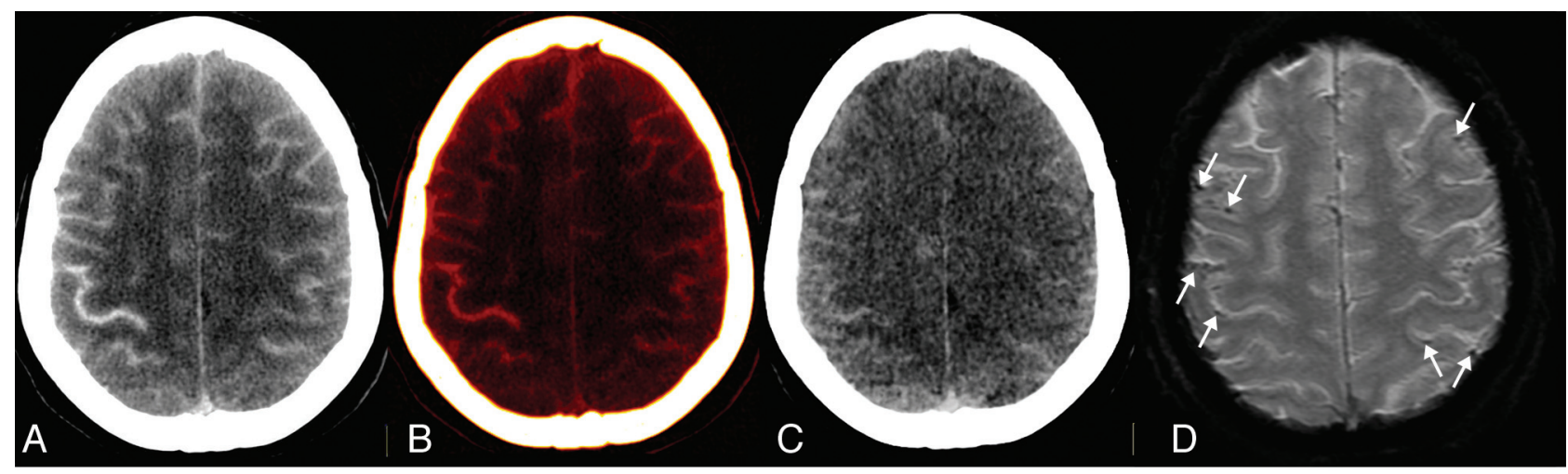

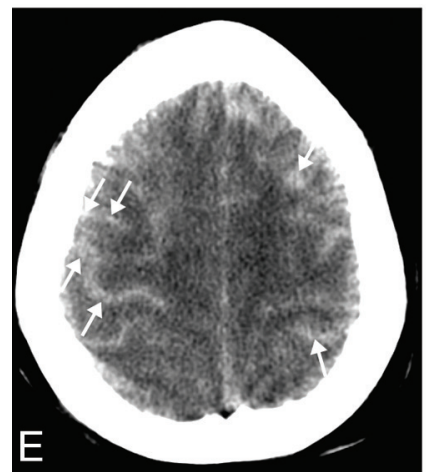

Fig 5. Subarachnoid hyperattenuation due to mixed contrast and hemorrhage in a 65-year-old woman treated for an acute stroke in the left MCA territory. Diffuse sulcal hyperattenuation in bilateral cerebral hemispheres is seen on SE image $(A)$ and iodine overlay image $(B)$. There are scattered areas of subarachnoid hyperattenuation on the VNC image $(C)$, likely representing superimposed hemorrhage. $D$, These areas of hemorrhage are confirmed on follow-up gradient-echo $\mathrm{T} 2^{*}$-weighted MR imaging as areas of decreased signal intensity (arrows).

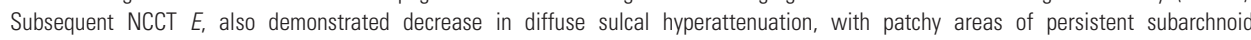
hyperattenuation (arrows). hemorrhage using VNC images from DECT angiography in 25 patients and compared image quality and CNR between VNC images from DECT angiography and single-energy NCCT. The VNC image quality was deemed to be sufficient in $96 \%$ of the cases. The assessment of intracranial hemorrhage agreed with the conventional unenhanced images $96 \%$ of the time on a lesion-by-lesion basis, and $100 \%$ of the time when compared on a patient-by-patient basis. The average CNR reached 2.63 in VNC images and 3.27 in conventional images. The authors concluded that the VNC images, though slightly noisier, are sufficient for the detection of intracranial hemorrhage.

Currently, identification of extravascular contrast requires serial imaging that demonstrates early washout (within 24-72 hours) of the hyperattenuated lesion. ${ }^{1,9-11}$ Hemorrhage is persistent over several days to weeks. This early period of diagnostic uncertainty has important implications for clinical decision making and may delay optimal medical management. ${ }^{2}$ Antithrombotic medication is commonly used in certain settings after endovascular stroke therapy. Anticoagulation may be employed to prevent recurrent embolization when there is cervical vessel stenosis or dissection. Antiplatelet therapy may be required to prevent in situ thrombosis after stent implantation. The presence or absence of ICH alters the risk-benefit ratio of these therapies.

Rapid diagnosis of ICH may be especially important in the management of acute stroke because significant ICH tends to occur relatively early following reperfusion therapy. ${ }^{12}$ In the National Institute of Neurological Disorders and Stroke tPA trial, $80 \%$ of fatal hemorrhages occurred within 12 hours of tPA administration; the remainder occurred within 24 hours. ${ }^{13}$ A study by Jang et $\mathrm{al}^{9}$ demonstrated subsequent development of significant hemorrhagic transformation in 6 of 31 (19.4\%) hyperattenuated lesions identified immediately after IAT, suggesting an ongoing evolution of ICH in the early posttreatment period. This further suggests that, in a proportion of cases, there is an opportunity to potentially limit hemorrhage growth.

The tissue characterization capabilities of DECT have been studied in different clinical applications, such as bone removal, virtual noncontrast imaging, myocardial and pulmonary perfusion, carotid plaque assessment, renal stone characterization, and visualization of tophaceous gout. ${ }^{3,4,14-20}$ The results presented here confirm the findings of recent studies ${ }^{4,5}$ that demonstrated that iodine could be effectively subtracted from a DECT angiogram to yield a virtual noncontrast image that rivaled traditional NCCT in its diagnostic utility for hemorrhage detection. Our study expands on these findings by demonstrating the ability of DECT to classify regions where iodine and hemorrhage may both be present. It also assesses the effectiveness of DECT in all intracranial compartments.

It should be noted that, in certain situations, the presence of iodinated contrast can be established with confidence, even on SE CT scans. For example, if the attenuation markedly exceeds that expected for hemorrhage (eg, values greater than $120 \mathrm{HU}$ ), it can be confidently assumed that there is a component of iodinated contrast. In this study, only a minority of the intraparenchymal hyperdensities ( 4 in the entire cohort) demonstrated such high attenuation levels. This finding, however, only confirms the presence of contrast-an associated hemorrhage cannot be excluded on single-energy CT scans. Decomposition into VNC and iodine overlay images using DECT overcomes this limitation. In fact, 1 of the 4 foci that exceeded $120 \mathrm{HU}$ attenuation was correctly identified by DECT as having an associated hemorrhagic component associated with contrast extravasation.

This study shows that subarachnoid extravasation of contrast after intra-arterial therapy is not uncommon. Thirtyseven hyperattenuated lesions were identified in the subarach- 
noid space. While there is redistribution of both hemorrhage and contrast in the subarachnoid space, contrast, which is water soluble, washes out much more quickly in the subarachnoid and intraventricular spaces when compared with hemorrhage. Persistence of a hyperattenuation in the subarachnoid space on 24- to 48-hour CT, therefore, confirms it as hemorrhage. Of the 37 lesions, 6 persisted and were deemed pure hemorrhage. An additional 11 lesions showed considerable washout in portions, while other portions persisted. These were thought to be a combination of hemorrhage and contrast. The remaining 20 lesions completely disappeared on the follow-up CT and were considered to be pure contrast extravasation. A case could be made that some of these 20 lesions represented small foci of hemorrhages that redistributed on subsequent images. However, considering the attenuation of the lesion on the first image, none of these lesions would have been expected to disappear if they had any hemorrhagic component. All these facts were corroborated by DECT.

The current study also points to a limitation of the 3-material discrimination (brain parenchyma, hemorrhage, and iodine) employed by DECT. DECT is unable to properly account for calcifications that are reflected on both the VNC and iodine overlay images. Calcification, therefore, is a confounder when discriminating ICH from iodinated contrast. Fortunately, in most cases, prior imaging and a telltale morphologic signature of calcification generally lends itself to correct classification even on SECT.

\section{Conclusions}

DECT has near-perfect accuracy in distinguishing intracranial hemorrhage from iodinated contrast extravasation or staining, and may be particularly helpful in patients who have recently undergone intra-arterial stroke therapy. This technique has some advantages over gradient-echo brain MR imaging, including a shorter acquisition time, lower cost, and lack of contraindications in patients with metallic implants. Our results demonstrate that DECT can play a key role in the management of acute ischemic stroke because hemorrhagic transformation, a major complication of reperfusion therapy, can be reliably detected using this technique.

Disclosures: Joshua Hirsch—UNRELATED: Consultancy: Phillips, CareFusion, Comments: Phillips involvement not directly related. Phillips is a multinational company focused on imaging. I participated in a focus group regarding $\mathrm{NI}$ practice for which I was given an honorarium. I would imagine (though I am not certain) that Phillips makes some sort of Dual Energy Product. CareFusion manufactures products for vertebral augmentation; participated in NextGen team; Royalties: CareFusion; Stock/Stock Options: IntraTech, NFocus, Nevro, Comments: IntraTech is a development stage company for ischemic stroke. NFocus is a development stage company for hemorrhagic stroke. Nevro is a development stage company for nerve imaging. Raul Nogueira-UNRELATED: Board Membership: Concentric Medical, eV3 Neurovascular, Coaxia, Neurointervention. Rajiv Gupta-UNRELATED: Payment for Lectures (including service on speakers bureaus): Siemens Erlangen, Comments: Rajiv Gupta presented a talk titled "Neuro Applications of Dual Energy CT" at Somatom World Conference, sponsored by Siemens Medical Solutions.

\section{References}

1. Mericle RA, Lopes DK, Fronckowiak MD, et al. A grading scale to predict outcomes after intra-arterial thrombolysis for stroke complicated by contrast extravasation. Neurosurgery 2000;46:1307-14; discussion 1314-15

2. Greer DM, Koroshetz WJ, Cullen S, et al. Magnetic resonance imaging improves detection of intracerebral hemorrhage over computed tomography after intra-arterial thrombolysis. Stroke 2004;35:491-95

3. Graser A, Johnson TR, Chandarana H, et al. Dual energy CT: preliminary observations and potential clinical applications in the abdomen. Eur Radiol 2009;19:13-23

4. Ferda J, Novak M, Mirka H, et al. The assessment of intracranial bleeding with virtual unenhanced imaging by means of dual-energy CT angiography. Eur Radiol 2009;19:2518-22

5. Gupta R, Phan CM, Leidecker C, et al. Evaluation of dual-energy CT for differentiating intracerebral hemorrhage from iodinated contrast material staining. Radiology 2010;257:205-11

6. Alvarez RE, Macovski A. Energy-selective reconstructions in $\mathrm{x}$-ray computerized tomography. Phys Med Biol 1976;21:733-44

7. Johnson TR, Nikolaou K, Wintersperger BJ, et al. Dual-source CT cardiac imaging: initial experience. Eur Radiol 2006;16:1409-15

8. Petersilka M, Bruder H, Krauss B, et al. Technical principles of dual source CT. Eur J Radiol 2008;68:362-68

9. Jang YM, Lee DH, Kim HS, et al. The fate of high-density lesions on the noncontrast CT obtained immediately after intra-arterial thrombolysis in ischemic stroke patients. Korean J Radiol 2006;7:221-28

10. Nakano S, Iseda T, Yoneyama T, et al. Early CT signs in patients with acute middle cerebral artery occlusion: incidence of contrast staining and haemorrhagic transformations after intra-arterial reperfusion therapy. Clin Radio 2006;61:156-62

11. Yoon W, Seo JJ, Kim JK, et al. Contrast enhancement and contrast extravasation on computed tomography after intra-arterial thrombolysis in patients with acute ischemic stroke. Stroke 2004;35:876-81

12. Khatri P, Wechsler LR, Broderick JP. Intracranial hemorrhage associated with revascularization therapies. Stroke 2007;38:431-40

13. Intracerebral hemorrhage after intravenous $t-P A$ therapy for ischemic stroke. The NINDS t-PA Stroke Study Group. Stroke 1997;28:2109-18

14. Graser A, Johnson TR, Hecht EM, et al. Dual-energy CT in patients suspected of having renal masses: can virtual nonenhanced images replace true nonenhanced images? Radiology 2009;252:433-40

15. Ruzsics B, Lee H, Zwerner PL, et al. Dual-energy CT of the heart for diagnosing coronary artery stenosis and myocardial ischemia-initial experience. Eur Radiol 2008; 18:2414-24

16. Thieme SF, Johnson TR, Lee C, et al. Dual-energy CT for the assessment of contrast material distribution in the pulmonary parenchyma. AJR Am J Roentgenol 2009; 193:144-49

17. Chandarana H, Godoy MC, Vlahos I, et al. Abdominal aorta: evaluation with dual-source dual-energy multidetector CT after endovascular repair of aneurysms-initial observations. Radiology 2008;249:692-700

18. Stolzmann P, Scheffel H, Rentsch K, et al. Dual-energy computed tomography for the differentiation of uric acid stones: ex vivo performance evaluation. Urol Res 2008;36:133-38

19. Lell MM, Hinkmann F, Nkenke E, et al. Dual energy CTA of the supraaortic arteries: technical improvements with a novel dual source CT system. Eur J Radiol 2009

20. Choi HK, Al-Arfaj AM, Eftekhari A, et al. Dual energy computed tomography in tophaceous gout. Ann Rheum Dis 2009;68:1609-12 